Draft Re-Submitted to ApJ on June 26, 2021

Preprint typeset using $\mathrm{LAT}_{\mathrm{E}} \mathrm{X}$ style emulateapj v. 04/20/08

\title{
NEUTRON STAR RADIUS MEASUREMENT WITH THE QUIESCENT LOW-MASS X-RAY BINARY U24 IN NGC 6397
}

\author{
Sebastien Guillot ${ }^{1}$, Robert E. Rutledge ${ }^{1}$, Edward F. Brown ${ }^{2}$ \\ 1 Department of Physics, McGill University, 3600 rue University, Montreal, QC, H3A-2T8, Canada \\ ${ }^{2}$ Department of Phys. and Astr., Michigan State University, 3250 Biomed. Phys. Sci. Building, East Lansing, MI 48824-2320, USA \\ Draft re-submitted to ApJ on June 26, 2021
}

\begin{abstract}
This paper reports the spectral and timing analyses of the quiescent low-mass X-ray binary (qLMXB) U24 observed during five archived Chandra/ACIS exposures of the nearby globular cluster NGC 6397, for a total of $350 \mathrm{ks}$. We find that the X-ray flux and the parameters of the hydrogen atmosphere spectral model are consistent with those previously published for this source. On short timescales, we find no evidence of aperiodic intensity variability, with $90 \%$ confidence upper limits during five observations ranging between $<8.6 \% \mathrm{rms}$ and $<19 \% \mathrm{rms}$, in the $0.0001-0.1 \mathrm{~Hz}$ frequency range $(0.5-8.0 \mathrm{keV})$; and no evidence of periodic variability, with maximum observed powers in this frequency range having a chance probability of occurrence from a Poisson-deviated light curve in excess of $10 \%$. We also report the improved neutron star (NS) physical radius measurement, with statistical accuracy of the order of $\sim 10 \%: R_{\mathrm{NS}}=8.9_{-0.6}^{+0.9} \mathrm{~km}$ for $M_{\mathrm{NS}}=1.4 M_{\odot}$. Alternatively, we provide the confidence regions in mass-radius space as well as the best-fit projected radius $R_{\infty}=11.9_{-0.8}^{+1.0} \mathrm{~km}$, as seen by an observer at infinity. The best-fit effective temperature, $k T_{\text {eff }}=80_{-5}^{+4} \mathrm{eV}$, is used to estimate the neutron star core temperature which falls in the range $T_{\text {core }}=(3.0-9.8) \times 10^{7} \mathrm{~K}$, depending on the atmosphere model considered. This makes U24 the third most precisely measured NS radius among qLMXBs, after those in $\omega$ Cen, and in M13.
\end{abstract}

Subject headings: stars: neutron — X-rays: binaries — globular clusters: individual (NGC 6397)

\section{INTRODUCTION}

The emission from low-mass X-ray binaries in quiescence (qLMXBs) is routinely studied to provide useful constraints on the physical models of the interior of neutron stars (NSs). The low luminosity $\left(10^{32}-10^{33} \mathrm{erg} \mathrm{s}^{-1}\right.$, 4-5 orders of magnitude lower than the outburst luminosities) of these objects was first observed in the post-outburst stages of the transient LMXBs Cen X4 and Aql X-1 (van Paradijs et al. 1987), and initially interpreted as a thermal blackbody emission powered by some low-level mass accretion onto the NS surface (Verbunt et al. 1994).

In an alternate explanation for the energy source of qLMXBs, the luminosity is provided by the energy released during accretion episodes by pressure-sensitive nuclear reactions (electron captures, neutron emission, and pycnonuclear reactions) in the NS deep crust. The theory of deep crustal heating (DCH; Brown et al. 1998) describes how the accreted matter piles up at the top of the NS surface, forcing the matter underneath to deeper layers of the crust, and how the energy is deposited in the crust. The resulting nuclear reaction chain releases $\sim 1.5 \mathrm{MeV}$ per accreted nucleon (see Gupta et al. (2007) and Haensel \& Zdunik (2008) for details about crustal heating models), and gives rise to a time-average luminosity directly proportional to the time-averaged accretion rate:

$$
\langle L\rangle=9 \times 10^{32} \frac{\langle\dot{M}\rangle}{10^{-11} M_{\odot} \mathrm{yr}^{-1}} \frac{Q}{1.5 \mathrm{MeV} \mathrm{amu}^{-1}} \mathrm{erg} \mathrm{s}^{-1}
$$

where $Q$ is the average heat deposited in the NS crust per accreted nucleon.

It was also suggested that the observed thermal spectrum of qLMXBs is the result of the energy deposited in the crust, heating the NS core during outbursts and thermally re-radiating away from the crust through the NS atmosphere on core-cooling timescales (Brown et al. 1998). It is thought that this atmosphere is composed of pure hydrogen since heavy elements gravitationally settle on short timescale ( seconds, Romani 1987; Bildsten et al. 1992) once the accretion from the low-mass evolved companion star onto the NS surface shuts off after an outburst. Models of NS H atmosphere (Raiagopal \& Romani 1996; Zavlin et al. 1996; McClintock et al. 2004; Heinke et al. 2006), now routinely used for qLMXBs, imply emission-area radii consistent with the entire area of the NS, compared to the $\lesssim 1 \mathrm{~km}$ emission-area radii in the previously imposed blackbody approximation (Rutledge et al. 1999). Spectral fitting of qLMXBs with such models also leads to the determination of the projected radius (as observed from infinity) defined by $R_{\infty}=R_{\mathrm{NS}} g_{\mathrm{r}}^{-1}=$ $R_{\mathrm{NS}}\left(1-2 G M_{\mathrm{NS}} / R_{\mathrm{NS}} c^{2}\right)^{-1 / 2}$, where $R_{\mathrm{NS}}$ is the physical radius of the NS.

In the spectra of some qLMXBs in the field of the Galaxy (for example Cen X-4, Rutledge et al. 2001b, and Aql X-1, Rutledge et al. 2001a), an additional powerlaw component, dominating the spectrum above $2 \mathrm{keV}$ and unrelated to the $\mathrm{H}$-atmosphere thermal emission, is observed. Proposed interpretations of this powerlaw tail include residual accretion onto the NS magnetosphere (Grindlay et al. 2001a; Cackett et al. 2005), 
shock emission via the emergence of a magnetic field (Campana \& Stella 2000), or an intrabinary shock between the winds from the NS and its companion star (Campana et al. 2004). However, recent analyses of the quiescent emission of LMXBs have shown that variations in the non-thermal component are correlated to the variations in the thermal component. This suggests the presence of a variable low-level accretion on the NS (for the LMXB XTE J1701-462, Fridriksson et al. 2010, and for the LMXB Cen X-4, Cackett et al. 2010).

Another characteristic of qLMXBs is the expected lack of strong variability on long or short timescales since their emission is dominated by the thermal radiation from the interior of the NS. While the thermal component is not expected to display intensity variability, other emission mechanisms (like those mentioned in the previous paragraph) may be responsible for intensity and spectral variations (Brown et al. 1998). However, recent outburst episodes can generate variations in the quiescent thermal luminosity on days to years timescales, as observed for the LMXB KS 1731-260 (Rutledge et al. 2002, following the models described in Ushomirsky \& Rutledge 2001, see also Brown \& Cumming 2009).

The DCH/H-atmosphere interpretation has been applied to a large number of historical transient LMXBs and provided $R_{\infty}$ measurements from high signal-tonoise $(\mathrm{S} / \mathrm{N})$ spectra. The following list is, to the best of our knowledge, exhaustive: 4U 1608-522 (Rutledge et al. 1999), 4U 2129+47 (Rutledge et al. 2000), Cen X-4 (Campana et al. 2000; Rutledge et al. 2001b), Aql X-1 (Rutledge et al. 2001a), KS 1731-260 (Rutledge et al. 2002), XTE J2123-058 (Tomsick et al. 2004), EXO 1747-214 (Tomsick et al. 2005), MXB 1659-29 (Cackett et al. 2006), 1M 1716-315 (Jonker et al. 2007a), 1H 1905+000 (Jonker et al. 2007b), 2S 1803-245 (Cornelisse et al. 2007), 4U 1730-22 (Tomsick et al. 2007), EXO 0748-676 (Degenaar et al. 2009), and XTE J1701-462 (Fridriksson et al. 2010).

However, the $10 \%-50 \%$ systematic uncertainty in the distances to qLMXBs in the field directly affects the $R_{\infty}$ measurement uncertainty. Obtaining precise constraints on the dense matter equation of state (EoS) is the observational motivation for measuring the radii of $\mathrm{NSs}$, requiring $\sim 5 \%$ accuracy on $R_{\infty}$ to be useful for this purpose (Lattimer \& Prakash 2004; Steiner et al. 2010). The known distances to globular clusters (GCs) and their expected overabundances of X-ray binaries (Hut et al. 1992) has motivated the search for qLMXBs in the core of GCs.

There are 26 GC qLMXBs spectrally identified so far (see Heinke et al. 2003 and Guillot et al. 2009 for two complementary lists). However, some of them have poorly constrained radius and temperature measurements. This can be due to low count statistics and/or a large amount of galactic absorption (for example $N_{\mathrm{H}}=$ $1.2 \times 10^{22}$ atoms $\mathrm{cm}^{-2}$ for the GC Terzan 5) in their direction which alters the low-energy end $(0.1-1 \mathrm{keV})$ of the spectra where the H-atmosphere spectrum of qLMXBs peaks. Therefore, their identification is regarded as less secure. Most qLMXBs in GCs have low-S/N spectra, and therefore, have rather large uncertainties on their $R_{\infty}$ measurements ( $\sim 15 \%$ or more), precluding their use for
TABLE 1

Chandra OBSERVATIONS OF NGC 6397

\begin{tabular}{rrrrr}
\hline \hline $\begin{array}{c}\text { Obs. } \\
\text { ID }\end{array}$ & $\begin{array}{c}\text { Starting } \\
\text { Time (TT) }\end{array}$ & $\begin{array}{c}\text { Exposure } \\
\text { Time (ks) }\end{array}$ & Detector & Mode \\
\hline 79 & 2000 Jul 31 15:31:33 & 48.34 & ACIS-I3 (FI) & F \\
2668 & 2002 May 13 19:17:40 & 28.10 & ACIS-S3 (BI) & F \\
2669 & 2002 May 15 18:53:27 & 26.66 & ACIS-S3 (BI) & F \\
7460 & 2007 Jul 16 06:21:36 & 149.61 & ACIS-S3 (BI) & VF \\
7461 & 2007 Jun 22 21:44:15 & 87.87 & ACIS-S3 (BI) & VF \\
& & & & \\
\hline
\end{tabular}

Note. - All observations were performed with a $3.24104 \mathrm{~s}$ frame rate. $\mathrm{TT}$ refers to terrestrial time. FI and BI refer to front-illuminated and back-illuminated detectors, respectively.

EoS constraints. So far, only a few qLMXBs (in $\omega$ Cen, Gendre et al. 2003a, in M13, Gendre et al. 2003b, and $\mathrm{X} 7$ in 47 Tuc, Heinke et al. 2006) have spectra with S/N high enough to provide useful constraints on the dense matter EoS (Webb \& Barret 2007; Steiner et al. 2010).

The close proximity of the globular cluster NGC 6397 $(d \approx 2.5 \mathrm{kpc}$, Harris 1996; Hansen et al. 2007; Strickler et al. 2009), the moderately low galactic ab$\operatorname{sorption}^{1}\left(N_{\mathrm{H}}=0.14 \times 10^{22}\right.$ atoms cm ${ }^{-2}$, noted $N_{\mathrm{H}, 22}=$ 0.14 hereafter) in its direction makes it a useful target for the spectral identification of qLMXBs. The discovery observation of the qLMXB U24 ${ }^{2}$ in NGC 6397 provided modest constraints on the NS projected radius: $R_{\infty}=4.9_{-1}^{+14} \mathrm{~km}$ (Grindlay et al. 2001b, Gr01 hereafter). The proximity of U24 to the GC core requires the use of the Chandra X-ray Observatory's angular resolution to positionally and spectrally separate the qLMXB from other sources in the crowded GC core. U24 lies at $d_{\mathrm{c}}=6.8 r_{\mathrm{c}} \approx 20^{\prime \prime}$ from the GC center (core radius $r_{\mathrm{c}}=0.05^{\prime}$ and half-mass radius $r_{\mathrm{HM}}=2.33^{\prime}$, NGC 6397 is a core-collapse cluster). The reported effective temperature of U24 was $k T_{\text {eff }}=57-92 \mathrm{eV}$ (90\% confidence interval, Gr01).

This paper presents the spectral and timing analyses of five archived deep Chandra-ACIS observations of U24, located in GC NGC 6397. These long exposures provide the high-S/N data necessary to confirm the qLMXB classification of U24 by obtaining precise $R_{\mathrm{NS}}$ estimation. The lack of variability also supports this classification. The organization of this paper is as follows. Section 2 describes the data reduction and the analyses. Section 3 presents the results of the analyses. Section 4 provides a discussion of the results and Section [5] is a short conclusion.

\section{DATA REDUCTION AND ANALYSIS}

\subsection{Observations, Source Detection, and Count Extraction}

We analyze one archived Chandra/ACIS-I and four archived Chandra/ACIS-S observations of NGC 6397 (Table 1). The source detection and the data analysis are performed using the CIAO v4.1.1 package (Fruscione et al. 2006). The event files (level=1) are reprocessed with the latest calibration files from CALDB v4.1 Graessle et al. 2007, with the latest effective area

1 From http://cxc.harvard.edu/toolkit/colden.jsp using the NRAO data (Dickey \& Lockman 1990).

2 The source name U24 was used in the discovery paper (Gr01) and will be used throughout this work. 
TABLE 2

Position of U24 in Chandra Observations of NGC 6397

\begin{tabular}{ccccccc}
\hline \hline Obs. ID & $\begin{array}{c}\text { R.A. } \\
(\mathrm{J} 2000)\end{array}$ & $\begin{array}{c}\Delta_{\mathrm{R} . \mathrm{A}} \\
\left({ }^{\prime \prime}\right)\end{array}$ & $\begin{array}{c}\text { Decl. } \\
(\mathrm{J} 2000)\end{array}$ & $\begin{array}{c}\Delta_{\text {Decl. }} \\
\left({ }^{\prime \prime}\right)\end{array}$ & $\begin{array}{c}\text { Detection } \\
\text { Significance }\end{array}$ & Reference \\
\hline 79 & $17^{\mathrm{h}} 40^{\mathrm{m}} 41.421^{\mathrm{s}}$ & 0.02 & $-53^{\circ} 40^{\prime} 04.73$ & 0.02 & - & Gr01 \\
79 & $17^{\mathrm{h}} 40^{\mathrm{m}} 41.459^{\mathrm{s}}$ & 0.6 & $-53^{\circ} 40^{\prime} 04.47$ & 0.6 & $128 \sigma$ & This work \\
2668 & $17^{\mathrm{h}} 40^{\mathrm{m}} 41.489^{\mathrm{s}}$ & 0.6 & $-53^{\circ} 40^{\prime} 04.38$ & 0.6 & $152 \sigma$ & This work \\
2669 & $17^{\mathrm{h}} 40^{\mathrm{m}} 41.485^{\mathrm{s}}$ & 0.6 & $-53^{\circ} 40^{\prime} 04.53$ & 0.6 & $149 \sigma$ & This work \\
7460 & $17^{\mathrm{h}} 40^{\mathrm{m}} 41.486^{\mathrm{s}}$ & 0.6 & $-53^{\circ} 40^{\prime} 04.60$ & 0.6 & $302 \sigma$ & This work \\
7461 & $17^{\mathrm{h}} 40^{\mathrm{m}} 41.488^{\mathrm{s}}$ & 0.6 & $-53^{\circ} 40^{\prime} 04.54$ & 0.6 & $228 \sigma$ & This work
\end{tabular}

Note. $-\Delta_{\text {R.A. and }} \Delta_{\text {Decl. }}$ are the uncertainties on the position, dominated by the Chandra systematic uncertainty in this work. The positions reported previously (Grindlay et al. 2001b, , noted Gr01) have been corrected for the Chandra systematic uncertainty (see details in Section 3.1.

maps, quantum efficiency maps, and gain maps), as recommended in the CIAO Analysis Thread "Reprocessing Data to Create a New Level=2 Event File" to include the up-to-date CTI corrections (charge-transfer inefficiency).

The re-processed event files are analyzed including counts in the $0.5-8.0 \mathrm{keV}$ energy range. The full-chip light curves do not show evidence of background flares in any of the five observations, allowing use of the full exposure time of each observation. The low flux of the source of interest, U24, allows neglecting pile-up; the count rate of $\sim 0.06$ photons per frame corresponds to a pile-up fraction of less than $2 \%^{3}$.

For each observation (ObsID), the source detection is performed with the CIAO wavdetect algorithm. An exposure map is created using the task mkexpmap prior to the source detection. The wavdetect exposure threshold expthresh is set to 0.1 and the wavelet detection scales are set to scales $=$ "1.02.0 4.0 8.0". Thirty-five sources are detected $(\sigma>3)$ on the ObsID 2668, 37 sources on ObsID 2669, 66 sources on ObsID 7460, 48 on ObsID 7461, and 37 on the ACIS-I observation ObsID 79.

This paper is solely focused on radius measurement and timing analysis on the qLMXB U24. While the source detection is performed over the whole ACIS chip, the following analysis pertains only to the source U24.

Counts are extracted with the CIAO script psextract around the source position in a circular region of radius $3^{\prime \prime}$, which ensures that $98 \%$ of the enclosed energy fraction at $1 \mathrm{keV}$ is included ${ }^{4}$. The closest detected source, located at $10.6^{\prime \prime}$ distance from U24, has 21.6 counts (background subtracted) within $1.5^{\prime \prime}$. It contributes to $\ll 0.04$ contamination counts within the extraction radius of U24 (on the longest observation). The background is extracted from an annulus centered at the qLMXB position with inner radius $5^{\prime \prime}$, and outer radius $30^{\prime \prime}$. Other detected sources within the background annulus are excluded with a $5^{\prime \prime}$ radius region, which eliminate $99.8 \%$ of source counts in the background region. For the deepest observation (ObsID 7460), 15 counts from other sources are within the extracted background (which contains 6187 counts). In other words, these constraints ensure that $\sim 0.25 \%$ of the background counts are due to other sources. Finally, the extraction radius

\footnotetext{
3 Chandra Observatory Proposer Guide v12.0, Figure 6.18, December 2009

${ }^{4}$ Chandra Observatory Proposer Guide v12.0, Figure 6.7, December 2009
}

(containing $98 \%$ of the ECF) does not require to apply a correction to the flux.

Following the CIAO Science Thread "Creating ACIS RMFs with mkacisrmf", the response matrices files (RMFs) are recalculated prior to the spectral analysis since the RMFs obtained from psextract are not suited for ACIS observations with focal plane temperature of $120^{\circ} \mathrm{C}$ (the usual mkrmf command does not use the latest calibration available in the case of $-120^{\circ} \mathrm{C}$ ACIS imaging data). In addition, the ancillary response files (ARFs) are also recalculated using the energy grid of the newly obtained RMFs. Overall, the extracted spectra, together with the RMFs and ARFs, are used for the spectral analysis. In those spectra, the effect of background counts can be ignored. Indeed, in the worst case (for ObsID 7460 ), the number of expected background events accounts for $2.4 \%$ of the total number of counts in the extracted region (78.0 background counts out of a total of 3188 counts), so that the background is neglected for the spectral analysis.

\subsection{Spectral Analysis}

For each of the five observations, two spectral files are created, one with unbinned events (for fitting with the Cash-statistics, Cash 1979) and one with binning (for fitting with the $\chi^{2}$-statistics). For the latter, the bin width in the $0.5-1.5 \mathrm{keV}$ energy range matches the energy resolution of the ACIS-S3 chip, i.e., $\sim 0.15 \mathrm{keV}$. Above $1.5 \mathrm{keV}$, four wider bins $(0.3 \mathrm{keV}, 0.6 \mathrm{keV}$, and two $3 \mathrm{keV}$ wide spectral bins) are created. In some cases of low count statistics, the last 2 or 3 bins are grouped together to maintain a minimum of 20 counts per bin. The main criterion for the creation of the spectral bins is the energy resolution of the detector, but the 20 counts minimum is imposed to ensure approximate Gaussian uncertainty in each bin. Such a binning avoids an artificially small reduced- $\chi^{2}$, and conserves the validity of $\chi^{2}$-statistics.

Spectral fitting is performed with the software XSPEC v12.5.1 (Arnaud 1996) using the publicly available model of NS H-atmosphere nsatmos (McClintock et al. 2004; Heinke et al. 2006). The model assumes non-magnetic NSs and has been computed for a range of surface gravity $g=(0.1-10) \times 10^{14} \mathrm{~cm} \mathrm{~s}^{-2}$. For the normalization parameter, nsatmos uses the emitting fraction of the NS surface. It is kept fixed to unity in this work; in other words, the whole NS surface emits. The distance parameter is held fixed as well at the value of NGC 6397, 
$d=2.5 \mathrm{kpc}$ (Hansen et al. 2007; Strickler et al. 2009). The NS mass is assumed to be $1.4 M_{\odot}$. Finally, the galactic absorption is taken into account using the phabs multiplicative model, with $N_{\mathrm{H}}$, the hydrogen column density parameter, set to $N_{\mathrm{H}, 22}=0.14$. The errors on the bestfit parameters $\left(R_{\mathrm{NS}}, k T_{\text {eff }}\right)$ are calculated using the command error in XSPEC with $90 \%$ confidence or using the command steppar. Confidence contours in mass-radius space are obtained with the steppar command with both the mass and the radius as free parameters. The results of the spectral analysis are presented in Section 3.2 .

\subsection{Variability Analysis}

For each of the five observations, we perform two analyses to search for source variability on timescales shorter than the duration of the observations, and one analysis for timescales spanning the time between the first and last observations.

Power Density Spectrum (PDS). The data from each observation were binned into a discrete light curve, with time bin size equal to the time resolution used in the observation $(\Delta T=3.24104 \mathrm{~s})$. A standard fastFourier transform (FFT) of the discrete data into frequency space was produced (Press et al. 1995), using an open-source FFT algorithm (Frigo \& Johnson 1998). This produced a Fourier transform of the data, covering the frequency range $1 / T-0.15427 \mathrm{~Hz}$, where $T$ is the duration of the observation, and $0.15427 \mathrm{~Hz}$ is the Nyquist frequency, with discrete frequency resolution $1 / T$, and a total of $N / 2$ frequency bins, where $N=T / \Delta T$ is the number of time bins in the light curve. The resulting Fourier transform was then converted into a PDS, where the power $P_{j}$ in each frequency bin $j$ is $P_{j}=a_{j}^{2}+b_{j}^{2}$, where $a_{j}$ and $b_{j}$ are the real and imaginary parts of the Fourier component associated with a frequency $f_{j}=j / T$, producing a PDS. The data were then normalized according to a standard prescription for analyses (Leahy et al. 1983).

Short-Timescale ( $<1$ day) Variability. Shortterm variability in each ObsID is assessed by visual inspection of the five sources light curves. In addition, a Kolmogorov-Smirnov (K-S) test (Press et al. 1995) is performed to quantify the consistency of the temporal distribution of counts in each observation with a constant count rate. More specifically, the integrated-count light curve is compared to a linear distribution using a K-S test. A low K-S probability would indicate that the integrated light curve is significantly different from a linear distribution, demonstrating the presence of variability on the timescale of the observation.

Long-Timescale ( months-years) flux variability. Long-term variability is investigated to determine if the flux remained constant over the course of the five observations, between 2000 and 2007 . This is performed by adding a multiplicative constant parameter to the spectral model, and by fitting the five spectra simultaneously. The constant is kept fixed at a value $c=1$ for one spectrum (ObsID 79) and as a free and untied parameter for the remaining four spectra while the remaining spectral parameters $\left(N_{\mathrm{H}}, R_{\mathrm{NS}}, k T_{\text {eff }}\right)$ are assumed to be the same across all observations. Best-fit $c$ values statistically consistent with unity would demonstrate that the source flux remained constant on the timescale of the five observations.

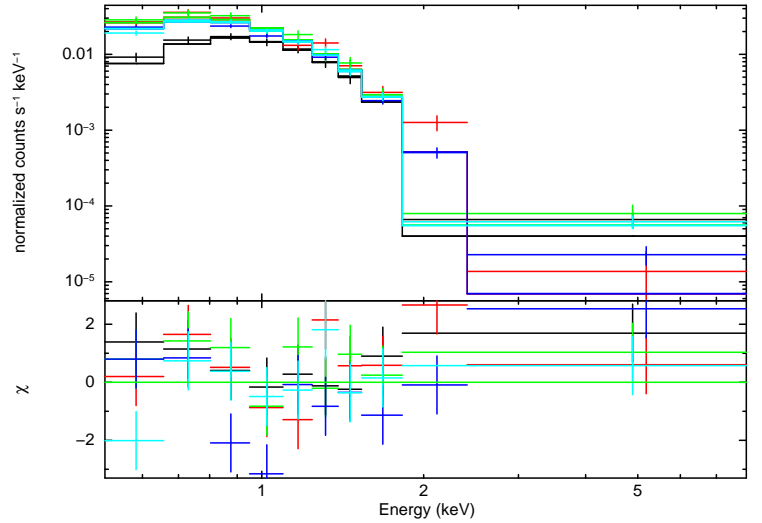

Fig. 1.- Folded spectra of all five Chandra-ACIS observations of U24 in NGC 6397, in the $0.5-8.0 \mathrm{keV}$ energy range. Each color corresponds to the spectra of each observations: ObsID 79 in black, ObsID 2668 in red, ObsID 2669 in green, ObsID 7460 in blue, ObsID 7461 in cyan blue [See the electronic edition of the paper for a color version of this figure]. The solid lines are the best-fitting model of an NS H-atmosphere model: NSATMOS, with $N_{\mathrm{H}, 22}=0.14$ and $M_{\mathrm{NS}}=1.4 M_{\odot}$ kept frozen. The spectral binning was performed in accordance with the Chandra energy resolution (see Section 2.2). The fit is statistically acceptable with $\chi_{\nu}^{2} /$ dof (prob.) $=$ $1.49 / 45(0.02)$. The residuals, shown in the lower part, indicate evidence of a count deficiency between $0.8 \mathrm{keV}$ and $1.2 \mathrm{keV}$ (see Figure 2 and Section 3.2), as well as an apparent excess of counts at large energy, above $2.5 \mathrm{keV}$, which is fit with a power-law component with $\alpha=1$ to estimate the contribution to the total flux (see Section 3.2)

\section{RESULTS}

\subsection{Positional Analysis}

The position of the source reported in the discovery observation (ObsID 79) is R.A. $=17^{h} 40^{m} 41.421^{s}$ and decl. $=-53^{\circ} 40^{\prime} 04.73^{\prime \prime}$ (Gr01). The authors corrected for the systematic Chandra uncertainty in the pointing $\left(0.6^{\prime \prime}\right)$ by cross-identifying cataclysmic variables on Chandra and Hubble Space Telescope (HST) observations. The positional uncertainty on the source is subarcseconds and consists in the residual error from the correction and the statistical uncertainty from the detection algorithm.

For comparison, the source detection in all five observations presented here led to the positions listed in Table 2 . It is concluded that the position of U24 is consistent with that reported previously, within the $0.6^{\prime \prime}$ Chandra systematic uncertainty. No correction for the systematic uncertainty is performed here since the identification of U24 is free of source confusion and since the purpose of this paper focuses on the spectral analysis.

\subsection{Spectral Analysis}

The spectral analysis is performed using the Cashstatistics on the unbinned data (neglecting the background) and using $\chi^{2}$-statistics on the grouped data. Results are presented in Table 3 listing the best-fit parameters for Cash and $\chi^{2}$-statistics, freezing and thawing $N_{\mathrm{H}}$. All reported errors are $90 \%$ confidence. The $\chi^{2}$-statistics null hypothesis probability confirms the viability of the fitted model while the Cash-statistics assumes that the model describes the data. Nonetheless, the use of Cashstatistics provides smaller uncertainties on the best-fit parameters, specifically for the NS radius measurements.

A simultaneous fit is also performed using all five spec- 
TABLE 3

Spectral Parameters of U24 in Chandra Observations of NGC 6397

\begin{tabular}{|c|c|c|c|c|c|c|}
\hline Obs. ID & $N_{\mathrm{H}, 22}$ & $R_{\mathrm{NS}}(\mathrm{km})$ & $k T_{\text {eff }}(\mathrm{eV})$ & $F_{\mathrm{X}}$ & Statistics & PL Contrib. \\
\hline \multirow[t]{4}{*}{79} & $(0.14)$ & $10.7_{-2.3}^{+3.3}$ & $73_{-10}^{+10}$ & $1.50_{-0.22}^{+0.14}$ & Cash: $256.7(100 \%)$ & - \\
\hline & $0.09_{-0.03}^{+0.03}$ & $6.8_{-1.8}^{+5.1^{3}}$ & $97_{-32}^{+30}$ & 1.27 & Cash: $254.2(0 \%)$ & - \\
\hline & $(0.14)$ & $10.1_{-5.1 p}^{+3.4}$ & $75_{-11}^{+49}$ & $1.49_{-0.22}^{+0.11}$ & $\chi_{\nu}^{2} /$ dof $($ prob. $)=0.76 / 7(0.62)$ & $\leq 8.8 \%$ \\
\hline & $0.10_{-0.03}^{+0.05}$ & $7.2_{-2.2 p}^{+3.2 .2 p}$ & $93_{-28}^{+42}$ & 1.30 & $\chi_{\nu}^{2} /$ dof $($ prob. $)=0.60 / 6(0.73)$ & - \\
\hline \multirow[t]{4}{*}{2668} & $(0.14)$ & $7.2_{-1.8}^{+2.4}$ & $95_{-19}^{+28}$ & $1.47_{-1.34}^{+20}$ & Cash: $213.3(100 \%)$ & - \\
\hline & $0.13_{-0.02}^{+0.03}$ & $7.1_{-1.6}^{+2.8}$ & $96_{-21}^{+26}$ & 1.44 & Cash: $213.1(0 \%)$ & - \\
\hline & $(0.14)$ & $7.8_{-2.8}^{+3.4}$ & $88_{-19}^{+43}$ & $1.44_{-5.81}^{+1.20}$ & $\chi_{\nu}^{2} /$ dof $($ prob. $)=1.81 / 9(0.61)$ & $\leq 3.9 \%$ \\
\hline & $0.13_{-0.03}^{+0.04}$ & $7.2_{-2.2}^{+4.6}$ & $94_{-27}^{+41}$ & 1.38 & $\chi_{\nu}^{2} /$ dof $($ prob. $)=1.97 / 8(0.46)$ & - \\
\hline \multirow[t]{4}{*}{2669} & $(0.14)$ & $10.0_{-5.0 p}^{+2.6}$ & $76_{-9}^{+37}$ & $1.52_{-0.26}^{+0.06}$ & Cash: $159.0(98 \%)$ & - \\
\hline & $0.11_{-0.02}^{+0.05}$ & $7.0_{-2.0 p}^{+5.0 p}$ & $96_{-32}^{+41}$ & 1.36 & Cash: $157.7(0 \%)$ & - \\
\hline & $(0.14)$ & $9.9_{-4.9 p}^{+3.0}$ & $77_{-10}^{+51}$ & $1.51_{-0.40}^{+0.05}$ & $\chi_{\nu}^{2} /$ dof $($ prob. $)=0.63 / 8(0.74)$ & $\leq 4.2 \%$ \\
\hline & $0.12_{-0.03}^{+0.07}$ & $7.2_{-2.2 p}^{+8.8}$ & $93_{-31}^{+44}$ & 1.37 & $\chi_{\nu}^{2} /$ dof $($ prob. $)=0.66 / 7(0.70)$ & - \\
\hline \multirow[t]{4}{*}{7460} & $(0.14)$ & $9.9_{-1.2}^{+1.2}$ & $75_{-5}^{+6}$ & $1.35_{-0.09}^{+0.04}$ & Cash: $367.0(100 \%)$ & - \\
\hline & $0.11_{-0.01}^{+0.01}$ & $6.9_{-0.9}^{+1.4}$ & $94_{-12}^{+13}$ & 1.18 & Cash: $355.1(0 \%)$ & - \\
\hline & $(0.14)$ & $10.1_{-1.5}^{+1.5}$ & $74_{-6}^{+7}$ & $1.35_{-0.17}^{+0.54}$ & $\chi_{\nu}^{2} /$ dof $($ prob. $)=2.32 / 8(0.02)$ & $\leq 4.7 \%$ \\
\hline & $0.11_{-0.01}^{+0.02}$ & $7.0_{-1.5}^{+1.9}$ & $93_{-21}^{+25}$ & 1.19 & $\chi_{\nu}^{2} /$ dof $($ prob. $)=2.14 / 7(0.04)$ & - \\
\hline \multirow[t]{4}{*}{7461} & $(0.14)$ & $6.7_{-1.0}^{+1.9}$ & $100_{-17}^{+19}$ & $1.37_{-1.19}^{+5.29}$ & Cash: $291.0(0 \%)$ & - \\
\hline & $0.14_{-0.02}^{+0.02}$ & $6.6_{-1.1}^{+2.6}$ & $101_{-24}^{+20}$ & 1.38 & Cash: $291.0(0 \%)$ & - \\
\hline & $(0.14)$ & $6.1_{-0.9}^{+3.1}$ & $104_{-31}^{+24}$ & $1.37_{-1.07}^{+1.33}$ & $\chi_{\nu}^{2} /$ dof $($ prob. $)=0.97 / 7(0.45)$ & $\leq 3.3 \%$ \\
\hline & $0.17_{-0.04}^{+0.06}$ & $10.7_{-5.7 p}^{+5.3}$ & $74_{-14}^{+52}$ & 1.57 & $\chi_{\nu}^{2} /$ dof $($ prob. $)=1.03 / 6(0.41)$ & - \\
\hline \multirow[t]{4}{*}{ All 5} & $(0.14)$ & $8.9_{-0.6}^{+0.9}$ & $80_{-5}^{+4}$ & $1.39_{-0.06}^{+0.02}$ & Cash: $1289.9(100 \%)$ & - \\
\hline & $0.12_{-0.01}^{+0.01}$ & $6.9_{-0.7}^{+1.0}$ & $95_{-10}^{+9}$ & 1.28 & Cash: $1276.1(69.2 \%)$ & - \\
\hline & $(0.14)$ & $9.3_{-1.0}^{+1.0}$ & $\begin{array}{c}78_{-4}^{+5} \\
7\end{array}$ & $1.39_{-0.06}^{+0.04}$ & $\chi_{\nu}^{2} /$ dof $($ prob. $)=1.49 / 45(0.02)$ & $\leq 3.7 \%$ \\
\hline & $0.12_{-0.01}^{+0.02}$ & $7.2_{-1.5}^{+1.9}$ & $92_{-14}^{+22}$ & 1.29 & $\chi_{\nu}^{2} /$ dof $($ prob. $)=1.16 / 44(0.03)$ & - \\
\hline
\end{tabular}

NOTE. - The model used for all spectral fits presented here is the NS H-atmosphere model NSATMOS, with the distance to the source fixed at the value $d=2.5 \mathrm{kpc}$ and the mass of the NS fixed at $M_{\mathrm{NS}}=1.4 M_{\odot} \cdot N_{\mathrm{H}, 22}$ is the galactic absorption $N_{\mathrm{H}}$ in units of $10^{22}$ atoms $\mathrm{cm}^{-2}$. Values in parentheses are held fixed for the spectral fits and quoted uncertainty are $90 \%$ confidence. $F_{\mathrm{X}}$ is the unabsorbed flux in units of $10^{-13} \mathrm{erg} \mathrm{cm}^{-2} \mathrm{~s}^{-1}$ in the $0.5-10 \mathrm{keV}$ range. The uncertainties on the fluxes were only calculated for the fixed- $N_{\mathrm{H}}$ fits. For ObsID 7460 , the $\chi^{2}$ value greater than 2 prevents $X S P E C$ to directly estimate the error region. It was obtained using the command steppar. "PL contrib." refers to the upper limit of a power-law component contribution to the total flux (see Section 3.2 . The percent in parenthesis following the Cash-statistic value indicates the goodness-of-fit. The best fit parameters and the statistics information provided do not include the power-law component.

tra, therefore increasing the count statistics and providing better constraints on the best-fit parameters (Figure 11). Prior to that, the source long-term variability is inspected, as described in Section 2.3. The best-fit values (with Cash-statistic) for the multiplicative constants described in Section 2.3 are $c=1.02_{-0.10}^{+0.09}, c=1.04_{-0.10}^{+0.09}$, $c=0.92_{-0.07}^{+0.06}$, and $c=0.95_{-0.08}^{+0.07}$, for the observations ObsID 2668, ObsID 2669, ObsID 7460, and ObsID 7461 , respectively, and $c=1$ (fixed) for ObsID 79. All bestfit values are statistically consistent with unity (within $1.5 \sigma)$, indicating that the source flux did not vary on long-term timescale. This allows for simultaneous spectral fitting with the constant multiplier fixed at the value $c=1$ for all five spectra.

The upper limit of a power-law contribution to the total flux is also estimated. To do so, a power-law component with fixed photon index $\alpha=1$ is added to the NS atmosphere model and the flux of this component using the upper limit of the power-law normalization parameter is measured. It is found that the power-law contribution accounts for $\leq 3.7 \%$ of the total flux (90\% confidence upper limit), when estimated from the simultaneous fits. Upper limits on the contribution of a power-law component for the individual spectra are also indicated in Table 3 .

There is some evidence for a count deficiency between
0.8 and $1.2 \mathrm{keV}$ on ObsID 7460 given the assumed spectrum and instrument calibration (see Figure 2). We parameterize this apparent dip in the spectrum with a notch component. The best-fit notch central energy is $E=0.96_{-0.02}^{+0.03} \mathrm{keV}$, and the best-fit width is $W=42 \pm 17 \mathrm{eV}$. The added component improves the statistics, $\chi_{\nu}^{2} /$ dof (prob.) $=1.35 / 6(0.23)$, without altering the best fit NS temperature and radius.

We also investigate the statistical significance of this deficiency. Using the method described in a previous work (Rutledge \& Sako 2003), we estimate the probability of observing such a deviation from a continuum model. First, the observed spectrum is convolved with the energy redistribution of the ACIS-S detector. Then, using a Monte Carlo approach, it is shown that the convolved spectrum does not exceed the $99 \%$ confidence limits envelope obtained from 10,000 simulated spectra (Figure 3). They were created using the best-fit nsatmos model $\left(R_{\mathrm{NS}}=8.9 \mathrm{~km}, k T_{\mathrm{eff}}=80 \mathrm{eV}, M_{\mathrm{NS}}=1.4\right.$, $d=2.5 \mathrm{kpc}$, and $N_{\mathrm{H}, 22}=0.14$, see Table 3). The maximum deviation corresponds to a $98.2 \%$ confidence, which does not constitute sufficient evidence to claim the detection of an absorption line, but which should be investigated in more details with higher $\mathrm{S} / \mathrm{N}$ observations.

For completeness, the results of spectral fits with other models are provided. Using an absorbed simple black- 
body model, the fit to the spectra (all five binned spectra) is not statistically acceptable: $\chi_{\nu}^{2} /$ dof (prob.) $=$ $3.51 / 45\left(2 \times 10^{-14}\right)$ with $N_{\mathrm{H}, 22}=0.14$ fixed, and $\chi_{\nu}^{2} /$ dof (prob.) $=1.631 / 44\left(5 \times 10^{-3}\right)$ with $N_{\mathrm{H}, 22}$ allowed to vary. A thermal bremsstrahlung model is also fit to the spectra, leading to a statistically acceptable fit $\left(\chi_{\nu}^{2} /\right.$ dof (prob.) $=$ $1.45 / 45(0.03))$ with best-fit parameter $k T=391 \pm 10 \mathrm{eV}$.

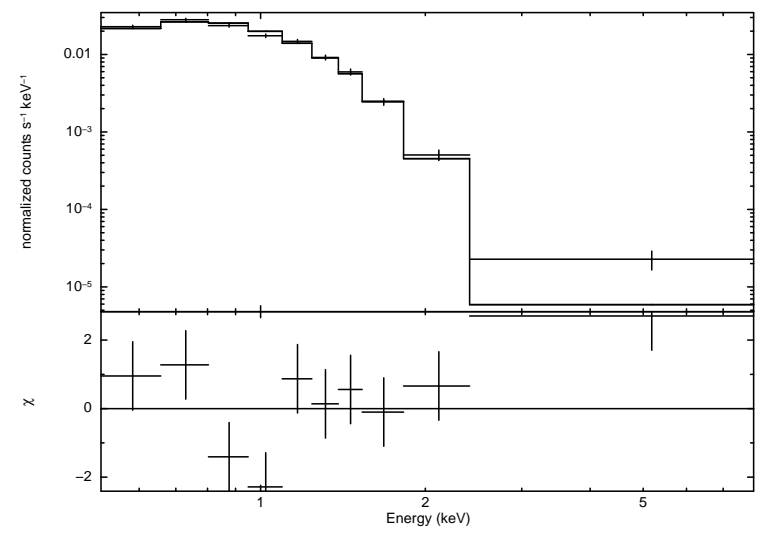

FIG. 2.- Folded spectrum of the ACIS observation 7460. As for Figure 1 the solid line shows the best-fitting NSATMOS model. The count deficiency observed between $0.8 \mathrm{keV}$ and $1.2 \mathrm{keV}$ does not exceed the $99 \%$ limit from the MC simulations of 10,000 spectra. In other words, the detection is not significant enough to claim the presence of an absorption line (see Section 3.2).

\subsection{Variability Analyses}

We find no evidence of broadband variability as a function of frequency in the PDS. For broadband variability, uncertainties were assigned to each frequency bin equal to the square root of the theoretical variance in the power, appropriate to the assumed normalization (Leahy et al. 1983), and derived $90 \%$ confidence $(2 \sigma)$ upper-limits on the root-mean-square ( $\mathrm{rms}$ ) variability. The data were then rebinned logarithmically and the resulting PDS were fit with a model of a constant power ${ }^{5}$, plus a power-law component in which the power scales $P_{j} \propto f_{j}^{-\alpha}$, and the power-law slope was held fixed at $\alpha=1$. This model was fit to the data using a Levenberg-Marquardt $\chi^{2}$ minimization technique (Press et al. 1995), to find the best-fit model parameters. The resulting 90\% confidence upper limits on the broadband variability, in a frequency range 0.0001$0.10 \mathrm{~Hz}$ (used for each observation, to ease comparison of limits, although the longer observations are sensitive to variability at frequencies below this range) and across the full Chandra/ACIS energy range $(0.5-8.0 \mathrm{keV})$ were $:<12 \%$ (ObsID 79), $\chi_{\nu}^{2}=0.63$ (26 dof) $<<19 \%$ (ObsID 2668), $\chi_{\nu}^{2}=1.5$ (27 dof) $;<11 \%$ (ObsID 2669), $\chi_{\nu}^{2}=1.32$ (26 dof); $<6.4 \%$ (ObsID 7460), $\chi_{\nu}^{2}=0.98$ (15 dof); $<8.6 \%$ (ObsID 7461), $\chi_{\nu}^{2}=0.98$ (9 dof).

\footnotetext{
5 The constant accounts for the expected Poisson noise power in the PDS, which is approximately 2 in the PDS normalization used; however, we observed the Poisson level for each of the PDS to be suppressed, to a value of $\sim 1.9$, due to instrumental deadtime effects. To correct for this, on average, in the rms variability upper limits derived here, the measured rms limits were increased by a factor $\langle A\rangle / 2.0$, where $\langle A\rangle$ is the best-fit Poisson power level.
}

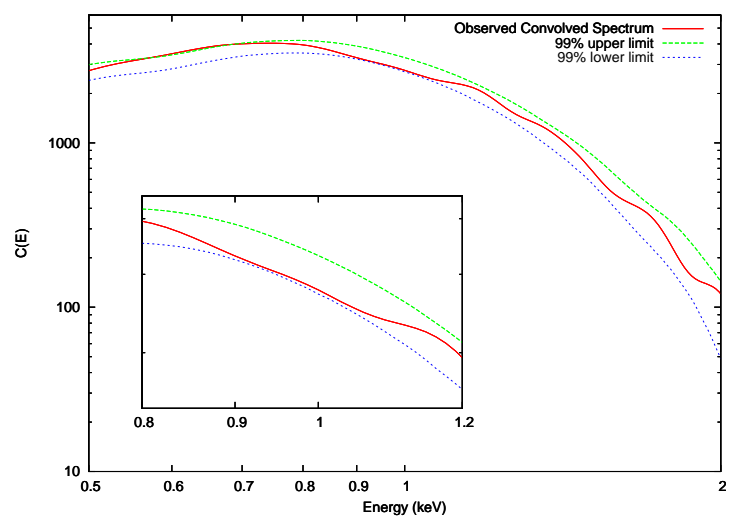

FIG. 3.- Results of the MC simulations analyzing the count deficiency observed in Figure 2 in the energy range $0.8-1.2 \mathrm{keV}$. The observed spectrum (solid line) convolved with the ACIS energy redistribution, $C(E)$, is shown within the $99 \%$ upper- and lowerlimit envelopes (dashed and dotted lines, respectively) obtained from Monte Carlo simulations (see Section 3.2).

We find no evidence of periodic variability. For ObsIDs $79,2668,2669,7460$, and 7461 respectively, we find maximum (normalized) powers of $P_{\max }=21.8,17.0,15.3$, 17.7 , and 23.7 which, with a number of frequency bins of $7526,4383,4154,23072$, and 13812 , correspond to respective probabilities of chance occurrence in all cases of $>10 \%$.

Visual inspection of the light curves did not reveal any variation in the source count rates. Short-term variability was also investigated in a more quantitative way by comparing the integrated ligthcurve with a linear distribution. None of the five observations showed an integrated light curve significantly different from a linear distribution. More specifically, the calculated K-S probabilities were $65 \%$ (ObsID 79), 62\% (ObsID 2668), 95\% (ObsID 2669), 51\% (ObsID 7460) and 16\% (ObsID 7461). Therefore, we find no evidence of intensity variability on the timescale of the integration times, i.e., $\lesssim 1$ day.

\section{DISCUSSION}

\section{1. $R_{\infty}$ Calculation}

Producing realistic constraints on the $\mathrm{dEoS}$ requires obtaining values of $R_{\infty}$ relying on as few assumptions as possible. Keeping the mass fixed for the model fitting is therefore not appropriate for that purpose. We estimate the value of $R_{\infty}$ by permitting $M_{\mathrm{NS}}$ to vary, and calculating the contours of constant model probability resulting from the fits in a mass-radius space (Figure 41). This is done using the steppar command in XSPEC. The $90 \%$ confidence regions of $R_{\mathrm{NS}}$ and $M_{\mathrm{NS}}$ are obtained from the $90 \%$-contour in the mass-radius space: $R_{\mathrm{NS}}=9.7_{-0.8}^{+0.9} \mathrm{~km}$ and $M_{\mathrm{NS}}=1.13_{-0.32}^{+0.47} M_{\odot}$. The best-fit value of the projected radius is therefore $R_{\infty}=11.9 \mathrm{~km}$. The calculation of the uncertainties is complicated by the fact that the distribution of $R_{\mathrm{NS}}$ and $M_{\mathrm{NS}}$ is not symmetric around the best-fit values (i.e., not Gaussian). Moreover, $R_{\mathrm{NS}}$ and $M_{\mathrm{NS}}$ are highly correlated, as shown by the crescent shape of the contour in M-R space (Figure 4). Therefore, the calculation of the uncertainties on $R_{\infty}$ using Gaussian normal error propagation is not valid. We describe two methods to obtain the uncertainty on $R_{\infty}$.

The projected radius and its uncertainties can be ob- 


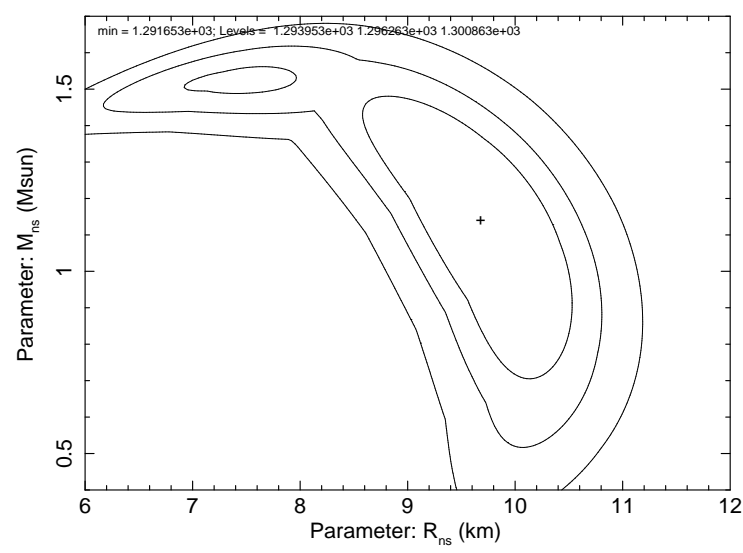

FIG. 4.- Contour plot in mass-radius space resulting from the simultaneous fit of the five data sets with the NSATMOS model. The $67 \%, 90 \%$, and $99 \%$ contours are shown, and the cross indicates the best fit (when both the mass and the radius are free to vary). For an NS mass of $1.4 M_{\odot}$, the range of $R_{\mathrm{NS}}$ corresponds to that obtained and quoted in Section 3.2

tained from a tabulated version of the nsa spectral model (Zavlin et al. 1996). However, this model (or nsa) is less adapted than the nsatmos or nsagrav models (Webb \& Barret 2007) because it was calculated for a single value of the surface gravity $g=2.43 \times 10^{14} \mathrm{~cm} \mathrm{~s}^{-2}$ while the other two models consider a range of values. Nevertheless, the best-fit $R_{\infty}$ value with this model is: $R_{\infty}=12.1_{-0.9}^{+1.5} \mathrm{~km}$ (consistent with the value calculated in the previous paragraph), for a temperature $k T_{\text {eff }}=76_{-3}^{+2} \mathrm{eV}\left(\chi_{\nu}^{2} /\right.$ dof (prob. $\left.)=1.54 / 44(0.02)\right)$.

A second method to estimate the uncertainties involves geometric construction, by reading graphically the error region of $R_{\infty}$ on the M-R contours (Figure 4). For that, we choose to use the line of constant surface gravity (i.e., constant $M(R)$ ) that goes through the point $(R, M)=\left(0 \mathrm{~km}, 0 M_{\odot}\right)$ and the point of best fit in M-R space. This line intersects the $90 \%$ contour at the points $(R, M)=\left(9.048 \mathrm{~km}, 1.045 M_{\odot}\right)$ and $(R, M)=\left(10.39 \mathrm{~km}, 1.245 M_{\odot}\right)$. These two points correspond to the values $R_{\infty}=11.15 \mathrm{~km}$ and $R_{\infty}=12.92 \mathrm{~km}$ which are, respectively, estimates of the lower and upper $90 \%$ confidence uncertainties on $R_{\infty}$, assuming a constant value of the surface gravity.

Therefore, the projected radius and its estimated $90 \%$ confidence uncertainties are: $R_{\infty}=11.9_{-0.8}^{+1.0} \mathrm{~km}$. With the achieved uncertainty, U24 becomes the third best radius measurement of a NS among the population of $\mathrm{GC}$ qLMXBs, after the ones in $\omega$ Cen (Gendre et al. 2003a) and in M13 (Gendre et al. 2003b).

\subsection{Error budget}

The high $\mathrm{S} / \mathrm{N}$ spectra and the precise radius measurements obtained in the work presented here can be used to constrain the EoS of dense matter. A high precision on the NS radius is mandatory to exclude some of the existing nuclear dense matter EoSs and provide the necessary constraints to understand the behavior of such matter. However, other sources of error come into play in this type of measurements. To quantify the total uncertainty on the radius measurement presented here, we estimate the contribution of each source of error into an error budget, including the distance to the GC NGC 6397, un- certainties intrinsic to the model used, systematic and statistical uncertainties. In those references where these uncertainties are discussed (for example, Heinke et al. 2006), only two of the three uncertainties we discuss here (distance and detector systematics) are addressed. No work that we can find in the literature discusses the impact of the uncertainty in the spectral model on derived model parameters; therefore, we do so here.

- The distance to the GC was recently measured using two independent methods. The analysis of the CO white dwarf (WD) sequence from deep observations in an outer field of NGC 6397 led to a distance of $2.54 \pm 0.07 \mathrm{kpc}$ (Hansen et al. 2007). More recently, using CO WDs in central regions of the cluster, the distance was calculated to be $2.34 \pm 0.13 \mathrm{kpc}$ (Strickler et al. 2009). The weighted mean of these two measurements is $2.50 \pm 0.06 \mathrm{kpc}$, corresponding to a $2.4 \%$ uncertainty. The unknown line-ofsight position of U24 within NGC 6397 accounts for $<0.1 \%$ of the distance uncertainty, which can be neglected compared to the GC distance uncertainty.

- The calculation of spectral model NSATMOS also contributes to the total uncertainty on the measured radius. However, the previous works describing the model do not provide a discussion on the fractional uncertainties in intensity due to convergence during the calculation of the spectral model (McClintock et al. 2004; Heinke et al. 2006). Therefore, it is not possible to evaluate the errors of the resulting spectra. The cited reference for similar models (NSA and NSAGRAV, Zavlin et al. 1996) only provides information on the temperature calculation convergence, which does not permit an estimation of the uncertainty error in the modeled intensity as a function of energy.

- The statistical uncertainties are those quoted in Table 3 (90\% confidence). This includes the 3\% systematic uncertainty of the detector calibration, taken into account using the "systematic 0.03" command in $X S P E C$.

Consequently, the distance uncertainty (2.4\%) is the only quantifiable error not taken into account in the radius measurement obtained from spectral fitting. It is therefore added in quadrature to the systematic and statistical uncertainties to obtain the total quantifiable uncertainty in the radius measurement. For example, the upper bound uncertainty limit of $R_{\mathrm{NS}}$ was $10.1 \%$ and is $10.4 \%$ when accounting for the distance uncertainty. The lower bound uncertainty limit was $6.7 \%$ and becomes $7.1 \%$. Consequently, the physical radius is $R_{\mathrm{NS}}=8.9_{-0.6}^{+0.9}$ (for $M_{\mathrm{NS}}=1.4 M_{\odot}$ ) while the estimated radiation radius is $R_{\infty}=11.9_{-0.8}^{+1.0} \mathrm{~km}$, when considering the sources of uncertainty listed above. In conclusion, in NGC 6397, the distance uncertainty of NGC 6397 alone minimally affects the current uncertainty on the radius measurement.

\subsection{Core temperature calculation}


The best-fit temperature and physical radius of the NS can be used to determine the interior temperature. This calculation is model dependent and due to the uncertainties in the deep atmosphere composition (the depth of the $\mathrm{H} / \mathrm{He}$ transition in particular), two different models are considered here. The first one assumes a layer of helium down to a column depth $y=1 \times 10^{9} \mathrm{~g} \mathrm{~cm}^{-2}$ with a pure layer of iron underneath. The second model considers a thin layer of He down to $y=1 \times 10^{4} \mathrm{~g} \mathrm{~cm}^{-2}$ with a mixture dominated by rp-processes. These two alternatives take into account the extremal values for the core temperature, for a fixed effective temperature.

The calculation, described in a previous work (Brown et al. 2002), was performed with a fixed mass $M_{\mathrm{NS}}=1.4 M_{\odot}$ and a fixed radius $R_{\mathrm{NS}}=8.9 \mathrm{~km}$, which corresponds to the best-fit value. The effective temperature used was $k T_{\text {eff }}=80_{-5}^{+4} \mathrm{eV}$. The calculation is performed down to a column depth of $1 \times 10^{14} \mathrm{~g} \mathrm{~cm}^{-2}$, since the temperature is nearly isothermal in deeper layers.

For the first model, the resulting interior temperature (at $\left.y=10^{14} \mathrm{~g} \mathrm{~cm}^{-2}\right)$ is $T_{\text {core }}=\left(3.37_{-0.41}^{+0.36}\right) \times 10^{7} \mathrm{~K}$. The second model leads to the value of interior temperature $T_{\text {core }}=\left(8.98_{-0.98}^{+0.81}\right) \times 10^{7} \mathrm{~K}$. Overall, if it is assumed that the $\mathrm{H} / \mathrm{He}$ transition depth is unknown, the core temperature is in the range of extreme values: $T_{\text {core }}=(3.0-9.8) \times 10^{7} \mathrm{~K}$.

\section{CONCLUSION}

We have performed the spectral analysis of five archived Chandra observations of the qLMXBs in the GC NGC 6397. The $\sim 350 \mathrm{ks}$ of integration time available permitted to obtain high $\mathrm{S} / \mathrm{N}$ spectra and improve the radius measurement. More specifically, the simultaneous spectral fitting of all five observations, using an NS H atmosphere, allowed us to provide constraints on the NS radius with $\sim 10 \%$ statistical uncertainty (90\% confidence). This confirmed the qLMXB nature of the X-ray source. Therefore, the measured NS properties are $R_{\mathrm{NS}}=$ $8.9_{-0.6}^{+0.9} \mathrm{~km}$ and $k T_{\text {eff }}=80_{-5}^{+4} \mathrm{eV}$, for $M_{\mathrm{NS}}=1.4 M_{\odot}$, and assuming an NS with an atmosphere composed of pure hydrogen. The estimated interior temperature lies in the range $T_{\text {core }}=(3.0-9.8) \times 10^{7} \mathrm{~K}$. In the $0.5-10 \mathrm{keV}$ range, the flux corresponding to these best-fit parameters is $F_{\mathrm{X}}=\left(1.39_{-0.06}^{+0.02}\right) \times 10^{-13} \mathrm{erg} \mathrm{cm}^{-2} \mathrm{~s}^{-1}$, equivalent to a luminosity of $L_{\mathrm{X}}=\left(1.04_{-0.05}^{+0.01}\right) \times 10^{32} \mathrm{erg} \mathrm{s}^{-1}$ at a distance of $2.5 \mathrm{kpc}$. The spectra did not show evidence for a power-law component as inferred by the upper limit on the contribution to the total flux of $3.7 \%$.

The results of this analysis were consistent with those of the discovery observation ( $\mathrm{Gr} 01)$; the reported NS radius and temperature were $R_{\infty}=4.9_{-1}^{+14} \mathrm{~km}$ and $k T_{\text {eff }}=57-92 \mathrm{eV}$. No optical counterpart was detected on the $H S T$ observations, with a limiting magnitude of $M_{V}>11$ ( $\left.\mathrm{Gr} 01\right)$.

No variability was observed on long timescales. Therefore, unless an outburst (for which there is no evidence) happened between the observations-between 2000 and 2002, or between 2002 and 2007-we conclude that the source remained in its quiescent stage since the discovery observation. It is worth noting that none of the GC qLMXBs discovered in quiescence so far have been seen in outburst. Moreover, an outburst happening between the available observations would have had an impact on the observed luminosity and intensity variability would have been detected (Ushomirsky \& Rutledge 2001; Rutledge et al. 2002). The search of shorttimescale variability ( $<1$ day) did not reveal any such variability. Finally, a PDS analysis was performed and did not demonstrate evidence of periodic variability in the frequency range $0.0001-0.10 \mathrm{~Hz}$. The lack of intensity variability on various timescales further supports the classification of the source.

In conclusion, the qLMXB U24 in the GC NGC 6397 adds to the list of qLMXBs suitable to place constraints on the dense matter EoSs with a best-fit projected radius of $R_{\infty}=11.9_{-0.8}^{+1.0} \mathrm{~km}$.

R.E.R. is supported by an NSERC Discovery grant. E.B. is supported by NASA/ATFP grant NNX08AG76G. The results presented have made use of data from archived observations available at the High Energy Astrophysics Archive Research Center Online Service, provided by the NASA GSFC. Finally, the authors would like to thank the referee for useful remarks that led to the improvement of this article.

\section{REFERENCES}

Arnaud, K. A. 1996, in Astronomical Society of the Pacific Conference Series, Vol. 101, Astronomical Data Analysis Software and Systems V, ed. G. H. Jacoby \& J. Barnes, 17-+

Bildsten, L., Salpeter, E. E., \& Wasserman, I. 1992, ApJ, 384, 143

Brown, E. F., Bildsten, L., \& Chang, P. 2002, ApJ, 574, 920

Brown, E. F., Bildsten, L., \& Rutledge, R. E. 1998, ApJ, 504, L95+

Brown, E. F. \& Cumming, A. 2009, ApJ, 698, 1020

Cackett, E. M., Brown, E. F., Miller, J. M., \& Wijnands, R. 2010, ApJ, 720, 1325

Cackett, E. M. et al. 2005, ApJ, 620, 922

Cackett, E. M., Wijnands, R., Linares, M., Miller, J. M., Homan, J., \& Lewin, W. H. G. 2006, MNRAS, 372, 479

Campana, S. et al. 2004, ApJ, 614, L49

Campana, S. \& Stella, L. 2000, ApJ, 541, 849

Campana, S., Stella, L., Mereghetti, S., \& Cremonesi, D. 2000, A\&A, 358, 583

Cash, W. 1979, ApJ, 228, 939

Cornelisse, R., Wijnands, R., \& Homan, J. 2007, MNRAS, 380, 1637

Degenaar, N. et al. 2009, MNRAS, 396, L26
Dickey, J. M. \& Lockman, F. J. 1990, ARA\&A, 28, 215

Fridriksson, J. K. et al. 2010, ApJ, 714, 270

Frigo, M. \& Johnson, S. G. 1998, in Acoustics, Speech and Signal Processing, 1998. Proceedings of the 1998 IEEE International Conference, in Seattle, WA, USA, ed. T. I. of Electrical \& N. J. Electronical Engineers, 1381-1384

Fruscione, A. et al. 2006, in Society of Photo-Optical

Instrumentation Engineers (SPIE) Conference Series, Vol. 6270, Society of Photo-Optical Instrumentation Engineers (SPIE) Conference Series, 62701V

Gendre, B., Barret, D., \& Webb, N. A. 2003a, A\&A, 400, 521

- 2003b, A\&A, 403, L11

Graessle, D. E., Evans, I. N., Glotfelty, K., He, X. H., Evans, J. D., Rots, A. H., Fabbiano, G., \& Brissenden, R. J. 2007, Chandra News, 14, 33

Grindlay, J. E., Heinke, C., Edmonds, P. D., \& Murray, S. S. 2001a, Science, 292, 2290

Grindlay, J. E., Heinke, C. O., Edmonds, P. D., Murray, S. S., \& Cool, A. M. 2001b, ApJ, 563, L53

Guillot, S., Rutledge, R. E., Bildsten, L., Brown, E. F., Pavlov, G. G., \& Zavlin, V. E. 2009, MNRAS, 392, 665 
Gupta, S., Brown, E. F., Schatz, H., Möller, P., \& Kratz, K.-L. 2007, ApJ, 662, 1188

Haensel, P. \& Zdunik, J. L. 2008, A\&A, 480, 459

Hansen, B. M. S. et al. 2007, ApJ, 671, 380

Harris, W. E. 1996, AJ, 112, 1487

Heinke, C. O., Grindlay, J. E., Lugger, P. M., Cohn, H. N., Edmonds, P. D., Lloyd, D. A., \& Cool, A. M. 2003, ApJ, 598, 501

Heinke, C. O., Rybicki, G. B., Narayan, R., \& Grindlay, J. E. 2006, ApJ, 644, 1090

Hut, P. et al. 1992, PASP, 104, 981

Jonker, P. G., Bassa, C. G., \& Wachter, S. 2007a, MNRAS, 377, 1295

Jonker, P. G., Steeghs, D., Chakrabarty, D., \& Juett, A. M. 2007b, ApJ, 665, L147

Lattimer, J. M. \& Prakash, M. 2004, Science, 304, 536

Leahy, D. A., Darbro, W., Elsner, R. F., Weisskopf, M. C., Kahn, S., Sutherland, P. G., \& Grindlay, J. E. 1983, ApJ, 266, 160

McClintock, J. E., Narayan, R., \& Rybicki, G. B. 2004, ApJ, 615, 402

Press, W., Flannery, B., Teukolsky, S., \& Vetterling, W. 1995, Numerical Recipies in C. The art of scientific computing (Cambridge University Press, Cambridge, UK)

Rajagopal, M. \& Romani, R. W. 1996, ApJ, 461, 327

Romani, R. W. 1987, ApJ, 313, 718

Rutledge, R. E., Bildsten, L., Brown, E. F., Pavlov, G. G., \& Zavlin, V. E. 1999, ApJ, 514, 945
- 2000, ApJ, 529, 985

- 2001a, ApJ, 559, 1054

- 2001b, ApJ, 551, 921

Rutledge, R. E., Bildsten, L., Brown, E. F., Pavlov, G. G., Zavlin,

V. E., \& Ushomirsky, G. 2002, ApJ, 580, 413

Rutledge, R. E. \& Sako, M. 2003, MNRAS, 339, 600

Steiner, A. W., Lattimer, J. M., \& Brown, E. F. 2010, ApJ, 722, 33

Strickler, R. R., Cool, A. M., Anderson, J., Cohn, H. N., Lugger, P. M., \& Serenelli, A. M. 2009, ApJ, 699, 40

Tomsick, J. A., Gelino, D. M., Halpern, J. P., \& Kaaret, P. 2004, ApJ, 610, 933

Tomsick, J. A., Gelino, D. M., \& Kaaret, P. 2005, ApJ, 635, 1233

- 2007, ApJ, 663, 461

Ushomirsky, G. \& Rutledge, R. E. 2001, MNRAS, 325, 1157

van Paradijs, J., Verbunt, F., Shafer, R. A., \& Arnaud, K. A. 1987, A\&A, 182, 47

Verbunt, F., Belloni, T., Johnston, H. M., van der Klis, M., \& Lewin, W. H. G. 1994, A\&A, 285, 903

Webb, N. A. \& Barret, D. 2007, ApJ, 671, 727

Zavlin, V. E., Pavlov, G. G., \& Shibanov, Y. A. 1996, A\&A, 315, 141 